\title{
Retention of mandibular advancement devices in the treatment of obstructive sleep apnea: an in vitro pilot study
}

\author{
Olivier M. Vanderveken • Paul Van de Heyning • \\ Marc J. Braem
}

Received: 29 November 2012 / Revised: 16 July 2013 / Accepted: 26 July 2013 / Published online: 21 August 2013

(C) The Author(s) 2013. This article is published with open access at Springerlink.com

\begin{abstract}
Purpose In order for a mandibular advancement device (MAD) to be efficacious, it must remain seated on the teeth during sleep. Quantitative data on the retentive characteristics of MADs are currently unavailable. The present pilot study is the first to describe an in vitro setup testing the retentive characteristics of different monobloc MADs.

Methods A hydraulic cyclic test machine was used with MADs seated on dental casts to measure retention forces upon removal of the MADs. A custom-made monobloc (CMmono), a thermoplastic monobloc (TP-mono), and a thermoplastic duobloc (TP-duo) configured as a monobloc were tested. Two protrusions were investigated, representing 25 and $65 \%$ of the maximal protrusion. The effects of the type of MAD, duration of the test, and amount of protrusion on removal forces were measured.

Results The measured removal forces of all three MADs tested differed significantly, with the TP-duo showing the highest values $(P<0.0001)$. The effects of wear due to the repetitive cyclic loading became obvious by the production of wear particles in all MADs tested. However, only the TP-duo showed a significant reduction in time in removal forces for
\end{abstract}

O. M. Vanderveken · P. Van de Heyning

Otolaryngology and Head and Neck Surgery,

University Hospital Antwerp, Edegem, Belgium

M. J. Braem

Lab Dental Materials, University of Antwerp, Campus

Groenenborger (CGB), Groenenborgerlaan 171, Wilrijk,

2020 Antwerp, Belgium

M. J. Braem ( $\square)$

Special Care Dentistry, University Hospital Antwerp,

Wilrijkstraat 10, 2650 Edegem, Belgium

e-mail: marc.braem@ua.ac.be

O. M. Vanderveken • P. Van de Heyning • M. J. Braem Faculty of Medicine and Health Sciences, University of Antwerp,

Antwerp, Belgium both protrusion positions $(P<0.0001 ; P=0.0011)$. The effect of the amount of protrusion on the removal forces differed significantly between all three MADs tested ( $P=0.0074)$. Conclusions This in vitro pilot study reveals significant differences in retention forces for the MADs tested. The findings are consistent with clinical effects of nightly loss of MADs as reported in the literature and are within the range of reported physiological mouth-opening forces. Future research is needed to determine the key design features of MADs that explain these differences.

Keywords Obstructive sleep apnea - Oral appliance design · Therapy $\cdot$ Retention $\cdot$ Oral appliance

\section{Introduction}

Obstructive sleep apnea (OSA), which affects $4 \%$ of men and $2 \%$ of women in the middle-aged population [1], increases the risk for cardiovascular diseases and traffic accidents [2]. An apnea-hypopnea index of at least five events per hour is the basis for an OSA diagnosis [3]. Oral appliance therapy with mandibular advancement devices (MADs) is the main alternative to continuous positive airway pressure (CPAP), the gold standard of treatment [4-8]. Both CPAP and MAD treatment have a beneficial effect on OSA-related cardiovascular morbidity $[2,9,10]$. Recently, in accordance with CPAP, an objective compliance measurement became also available for MAD therapy [11].

MADs advance the mandible and tongue, thereby improving upper airway patency during sleep and preventing upper airway collapse [12]. Although many types are available [13-16], all MADs require retention on the teeth to maintain protrusion of the mandible during sleep. Lack of retention causes loosening of the MAD and might result in reduced efficacy of OSA treatment, patient complaints about poor fit and an increased risk of side effects [15, 17, 18]. 
In vitro testing of the retentive characteristics of MADs could be considered as a first step in gaining more insight into the retention mechanisms involved and leading to the eventual definition of useful selection criteria for MADs. Therefore, the aim of this in vitro pilot study was to compare the retention forces upon removal of three different MAD designs: a custom-made monobloc, a thermoplastic monobloc, and a thermoplastic duobloc configured as a monobloc. The effects of the duration of the test and the amount of protrusion on the retention forces were also studied.

\section{Materials and methods}

A custom-made monobloc (CM-mono, University Hospital Antwerp, Belgium), a prefabricated thermoplastic monobloc (TP-mono, Somnoguard, TomeD Dr. Toussaint GmbH, Germany), and a prefabricated thermoplastic duobloc (TP-duo, Somnofit, Oscimed, Switzerland) were investigated. Dental impressions and casts were made (Impregum Pentasoft Medium, 3 M ESPE AG, Germany) for one patient with complete upper and lower dental arches including third molars and class II bite proportions. The CM-mono (Fig. 1a) was made in the dental lab using vacuum thermoforming techniques (Erkoflex, Erkodent Pfalzgrafenweiler, Germany). The upper part consisted of a single 4-mm-thick sheet (Erkoflex 581248). The lower part was multilayered with a 2-mm-thick inner sheet (Erkoflex 581258), a 1-mm-thick reinforcing intermediary sheet (Erkodur 521210), and a 4-mm-thick outer sheet (Erkoflex 581248). The margins were finished with a tungsten burr and heat-treated. Both the TP-mono (Fig. 1b) and TP-duo (Fig. 1c) were adapted chair-side by a dental sleep professional by softening the thermoplastic in a temperature-controlled hot water bath according to the respective manufacturers' instructions. The TP-duo was modified into a monobloc by melting both the upper and lower parts together to enable the experimental setup. Two protrusive positions were investigated: $3 \mathrm{~mm}(n=5$ appliances tested for CM-mono, TP-mono, TPduo) and $8 \mathrm{~mm}(n=3$ appliances tested for CM-mono; $n=5$ appliances tested for TP-mono and TP-duo), representing 25 and $65 \%$ of the maximal protrusion.

Fig. 1 Mounting of the custommade monoblock CM-mono (a), the thermoplastic monoblock TP-mono (b), and the thermoplastic duoblock TP-duo (c) on the epoxy casts
Epoxied casts were mounted in average value inclination of the occlusal plane (Fig. 2) and centered in a hydraulic cyclic test machine (Dartec HC10, testbench Dartec 9600 Controller, Dartec, UK). The forces required for removal of the MADs from these casts were continuously recorded during 730 cycles at $35^{\circ} \mathrm{C}$ in a dry environment to simulate 1 year of clinical use. A triangular loading/unloading wave curve $(7.5 \mathrm{~mm} / \mathrm{s}$ actuator speed) was applied. The stroke of the actuator head was linear for both protrusions and the length was set so that the MAD did not completely separate from the casts upon removal, preventing problems with misalignment at reinsertion during the next loading cycle. Statistical evaluation of the results was based on repeated measures ANOVA with three withinsubjects factors: MAD type, amount of protrusion and duration of test. All analyses were conducted using SAS 9.2 (SAS Institute, Cary, NC). Significance level was set at 0.05 .

\section{Results}

The measured removal forces of the three MADs tested differed significantly $(P<0.0001)$, with the TP-duo showing the highest values and the TP-mono showing the lowest (Table 1 and Fig. 3).

Table 1 shows the removal forces (in Newton) expressed as mean $( \pm \mathrm{SD})$ for both protrusion positions ( 3 and $8 \mathrm{~mm})$ at the first and the last cycle of the tests, revealing the effect of test duration: only the TP-duo showed a significant reduction in removal forces, which was true for both protrusion positions $(P<0.0001$ and $P=0.0011)$.

The effect of the amount of protrusion on the removal forces differed significantly between all three MADs tested $(P=0.0074)$ as shown in Fig. 3. The CM-mono showed higher retention forces at the $8-\mathrm{mm}$ protrusion compared to the $3-\mathrm{mm}$ position, both at the start and end of the test $(P<0.0001$ and $P=0.0003$, respectively). The TP-duo only showed higher retention forces at the start of the test in the $8-\mathrm{mm}$ protrusion compared to the 3 -mm protrusion $(P=0.0108)$.

Inspection of the inner surfaces of the tested MADs revealed the presence of wear particles and tear characteristics as shown in Fig. 4.
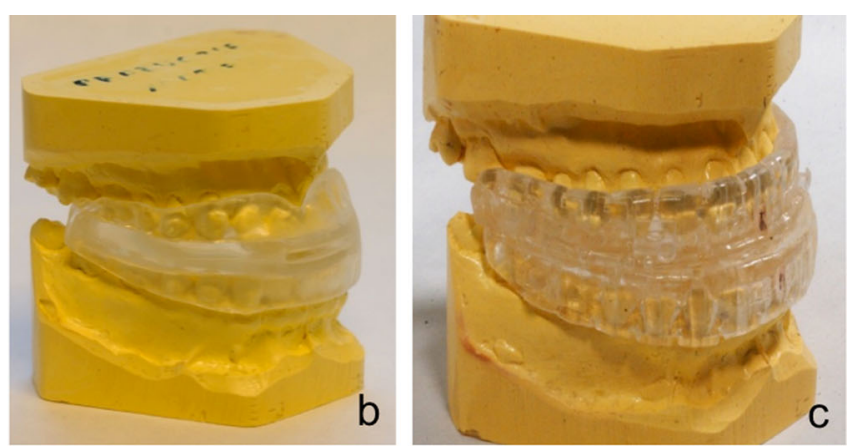

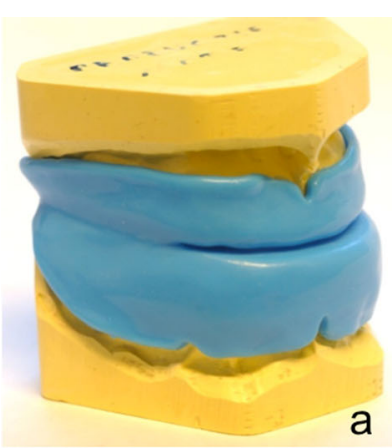




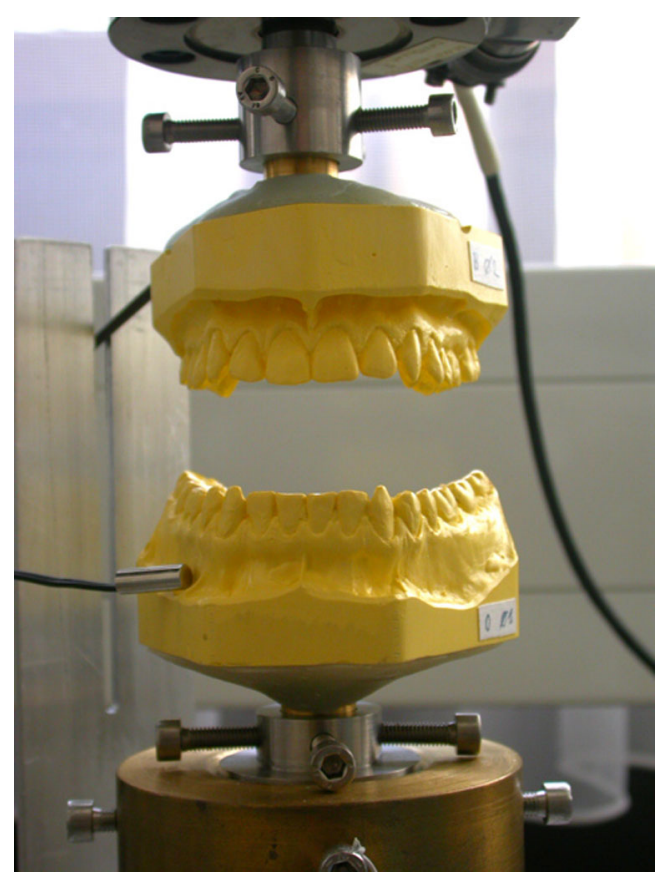

Fig. 2 Positioning of the epoxied models in the dynamic testing machine prior to insertion of the MAD. A temperature sensor is visible at the lower jaw

\section{Discussion}

In MAD therapy for OSA, a plethora of devices exist with different designs $[5,16,19]$. Such design features may influence the retention of the MAD, the degree of advancement of the mandible, and the range of movement of the mandible [19]. It is, however, unclear as to what extent some of these variations in design may affect clinical efficacy, adverse effects described by patients and patient therapy compliance $[19,20]$. It was reported that design features did not significantly influence OSA symptom severity, frequency, or duration [21]. Recent studies, however, suggest that devices designed to allow adjustment of mandibular protrusion are

Table 1 Removal forces $(\mathrm{N})$ expressed as mean $( \pm \mathrm{SD})$ for both protrusion positions ( 3 and $8 \mathrm{~mm}$ ) at the first and the last test cycle for the three types of MADs tested illustrating the effect of duration of the test

\begin{tabular}{llrrrrr} 
Type & $\begin{array}{l}\text { Protrusion, } \\
\mathrm{mm}\end{array}$ & Start (N) & $\mathrm{SD}$ & End (N) & $\mathrm{SD}$ & $P$ value* \\
\hline CM-mono & 3 & 17.6 & 2.2 & 22.0 & 1.2 & 0.0748 \\
& 8 & 38.7 & 4.0 & 35.7 & 0.6 & 0.3351 \\
TP-mono & 3 & 5.8 & 1.9 & 4.0 & 1.0 & 0.4535 \\
& 8 & 8.2 & 2.8 & 8.8 & 2.9 & 0.8017 \\
TP-duo & 3 & 40.6 & 12.3 & 31.8 & 6.1 & $0.0011^{\mathrm{a}}$ \\
& 8 & 48.0 & 9.7 & 32.6 & 9.4 & $<0.0001^{\mathrm{a}}$
\end{tabular}

${ }^{*} P$ value $=0.05$, significance level, result of the repeated measures ANOVA

${ }^{\text {a }}$ Statistically significant at the 0.05 probability level for the time effect more likely to provide successful therapy, especially in moderate to severe OSA, as compared to fixed monobloc appliances $[14,18]$. Design features may also influence retention of the MAD during sleep. Effective MAD therapy for OSA is thus complexly related to the degree of customization, the ability for titration of the mandible, as well as the retentive capacities of a particular MAD [13-15]. Knowledge of the retentive characteristics of a particular MAD is therefore essential for selection of a proper MAD for clinical use. To the best of the authors' knowledge, the present study is the first to use an in vitro setup to analyze and measure the retentive forces of different types of MADs. The results of the present study indicate that it is feasible to evaluate in vitro retentive characteristics of monobloc MADs.

The experiment design included the use of a hydraulic cyclic test machine and epoxied casts for measuring retention forces on removal of the MADs from the casts. As in any in vitro test, assumptions and compromises had to be made. First, the path of a removable denture is the direction in which it moves in relation to the supporting teeth when it is inserted or removed from its position, as guided by the contact of its rigid structures with the teeth. Interference structures must be detected and resolved in order to find a feasible path of insertion or removal [22]. Second, in vivo, some condylar rotation will occur before the mouth opens, followed by a translation on further opening as well as interaction with muscles that generate medio-lateral movements [23], which may also influence this path of removal. Such elements were not simulated in the present in vitro test, where a simplified straight line of removal was followed without fully respecting the path of least resistance. This could cause increased friction, resulting in higher removal forces in the present test than would occur clinically. Third, the tests were carried out in a dry environment, which could have had a dual effect: although it could have increased retention forces due to increased resistance, the absence of a lubricant also may have resulted in less optimal adaptation of the MAD due to a lack of contact between the inner layer of the MAD and the outer layer of the casts, thereby eliminating the adhesive layer that is formed in a completely removable denture. Thus, omitting lubrication also could have lowered the retentive forces being measured. Finally, the dental configuration of only one patient was tested; therefore, the influence of the inclination of teeth on the generated forces could neither be quantified nor compared with other dental configurations. This could be of importance regarding the retention forces with respect to the presence and magnitude of undercuts, as described in studies of the retention forces of thermoplastic clasps [24].

Nevertheless, the present setup does allow for a reproducible way of testing and shows a significant distinction between the measured removal forces of all three tested MADs. This finding emphasizes that classification of MADs in terms of construction materials cannot be translated directly into 
Fig. 3 Box plot showing the retention forces as a function of protrusion position. Plot showing the lower quartile, median, upper quartile, average (filled circle) and outliner (empty circle). The ends of the whiskers indicate the minimum and maximum of all the data

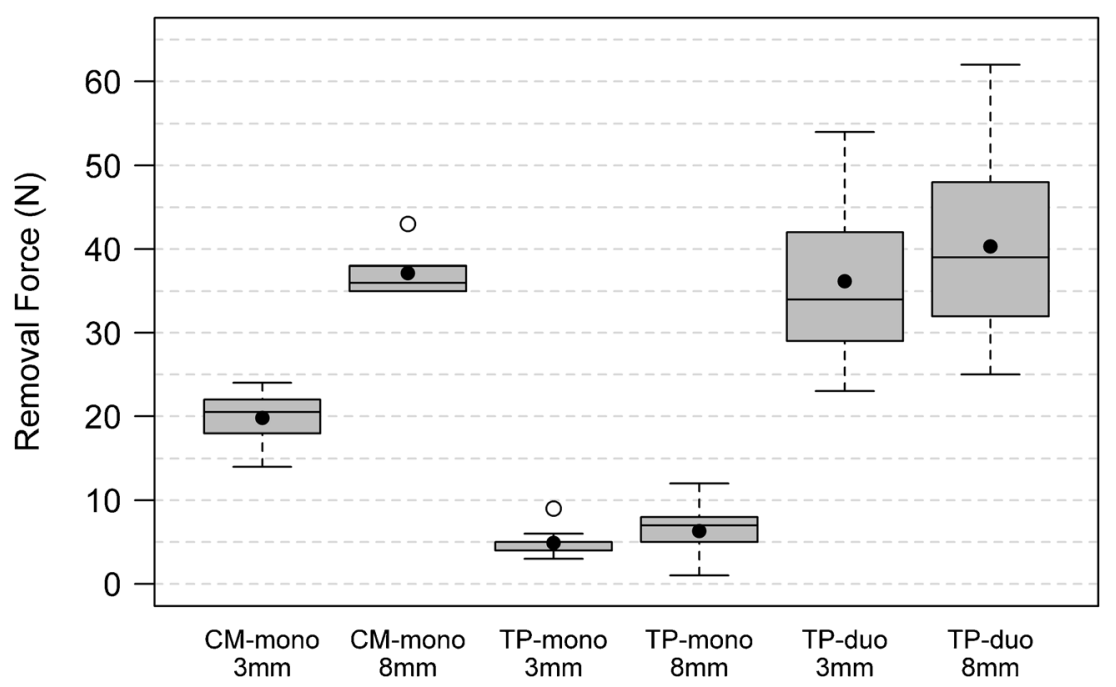

retentive behavior, as the TP-duo showed high retention forces compared to the TP-mono. Indeed, retention characteristics seem to be related to the application of, in this case, the thermoplastic. The thermoplastic "body" of the TP-duo is contained in a perforated rigid spoon to sustain the softened thermoplastic by mechanical retention upon cooling after adaptation. The higher removal forces found in the TP-duo as compared to the TPmono could be attributed to this design, which improves rigidity and fit. This conclusion supports recent findings with another prefabricated thermoplastic MAD (Somnoguard AP, TomeD Dr. Toussaint $\mathrm{GmbH}$, Germany) also contained in a rigid shell [18]. Finally, one may not expect a custom-made MAD like the CMmono to automatically guarantee the highest retention forces (Table 1 and Fig. 2) as compared to a chair-side adapted MAD, such as the TP-duo tested.

In the present study, it was found that retention forces could change significantly over time (Table 1), depending on the type of MAD. This is not surprising since wear will occur during repetitive insertion and removal of the MAD, causing a decrease in the degree of fit and thus lowering retention forces. Indeed, wear particles formed on all of the MADs tested as is shown in Fig. 4. The results indicate that the TP-duo suffered the most from wear effects, showing a significant decrease in retention forces over time. Although little is known about the effects of such wear patterns on MADs, it can be expected that general tribological principles will govern the materials' behavior under load [25], further emphasizing the importance of the materials chosen for constructing the MAD.

The results further show that an increased amount of protrusion seems to cause higher retention forces (Fig. 3). The present setup does not allow generalizing this finding since it might well depend on, e.g., the inclination of the upper incisors as well as other dental and experimental factors. It could however indicate that the retentive capacities of MADs might be related to the degree of mandibular protrusion. It is not yet known to what extent or how this can be translated into the clinic during, for example, titration procedures. Increased protrusion may cause the MAD to show higher retention forces, while at the same time, such forces might result in an increased risk of tooth displacement and side effects [26]. Furthermore, it is still unclear whether an increased amount of protrusion will maintain a linear relationship with therapeutic efficacy [26]. Additional research is needed to determine the effects of protrusion on retention.

An interesting approach could be to put the measured retention forces into clinical perspective. Jaw opening forces depend on the amount and speed of the opening movements [27]. At an interincisal distance of about $10 \mathrm{~mm}$, which is comparable to the average thickness of a MAD, pooled data showed that opening forces range from 2 to $9 \mathrm{~N}$ and increase substantially to an average force of $19.9 \pm 4.5 \mathrm{~N}$ at an interincisal distance of
Fig. 4 Wear particles and striae on the inner surfaces of the MADs tested: (left) CM-mono, (center) TP-mono, and (right) TP-duo
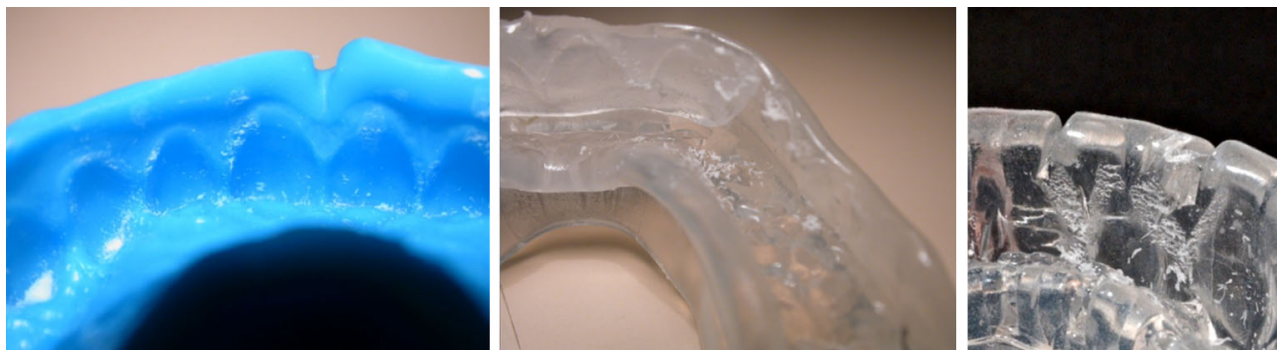
$49 \mathrm{~mm}$ at mouth opening. Looking at the magnitude of these literature values, it becomes clear that the TP-mono tested in the present in vitro setup with the particular jaw configuration does not possess sufficient retention forces to resist even initial jaw opening, let alone full mouth opening, whereas the other MADs tested could show sufficient retention forces. In clinical trials, loss of a MAD during sleep is often mentioned as one of the primary reasons for discontinuation of treatment $[15,18$, 28]. The present results support this finding and further substantiate the clinical data of Vanderveken et al. in which patients reported loss of a prefabricated adaptable thermoplastic MAD [15] during sleep, suggesting this could be explained by insufficient retention forces. Since the present results indicate that retention seems to be favored by a tight fit of the MAD onto the teeth, further research is required to describe the relationship between retention and reported side effects of thermoplastic appliances $[15,17,29]$. One must however realize that at this moment it is not yet clear what exactly the opening force is a MAD should resist. This answer is not yet available and can only be found in studying the individual patients' mouthopening forces in relation to the retentive capacity of a particular MAD. The present results do however tend to conclude that retentive forces that are just surpassing the passive opening of the mouth could be considered too low. Further research will need to address this topic.

No clinical data on the TP-duo are available yet to allow comparison of the present in vitro findings with in vivo data. However, a CM-mono similar in concept to the one tested in the present study has been evaluated clinically [15] with no complaints noted on loss of retention, emphasizing again the importance of a tight fitting MAD.

Retention could furthermore become of primary importance when the MAD is titrated towards its protrusive position of therapeutic effect. In a recent in vivo study [30] an almost linear relationship between recorded force and mandibular advancement was found, reaching up to $13.6 \mathrm{~N}$. Forces in this order of magnitude may not only cause potentially irreversible changes of occlusion [30] but may also increase the risk of loss of retention of the MAD. Further research is needed to translate the in vitro forces measured in the present test into clinically relevant indicators and to determine the key design features of MADs explaining the differences in retention characteristics measured.

Acknowledgments The devices were provided free of charge by TomeD GMBH and Oscimed Belgium, which the authors gratefully acknowledge. They thank Mr. Geert Keteleer for assistance in the testing of the MADs at the Lab Dental Materials, University of Antwerp, Wilrijk, Belgium. No other funding was obtained for the present project.

Conflict of interest The authors declare that they have no conflict of interest.

Open Access This article is distributed under the terms of the Creative Commons Attribution License which permits any use, distribution, and reproduction in any medium, provided the original author(s) and the source are credited.

\section{References}

1. Young T, Palta M, Dempsey J, Skatrud J, Weber S, Badr S (1993) The occurrence of sleep-disordered breathing among middle-aged adults. N Engl J Med 328(17):1230-1235

2. Vanderveken OM, Boudewyns A, Ni Q, Kashyap B, Verbraecken J, De Backer W, Van de Heyning P (2011) Cardiovascular implications in the treatment of obstructive sleep apnea. J Cardiovasc Transl Res 4(1):53-60

3. AASM (1999) Sleep-related breathing disorders in adults: recommendations for syndrome definition and measurement techniques in clinical research. The Report of an American Academy of Sleep Medicine Task Force. Sleep 22(5):667-689

4. Hoekema A, Doff MH, de Bont LG, van der Hoeven JH, Wijkstra PJ, Pasma HR, Stegenga B (2007) Predictors of obstructive sleep apneahypopnea treatment outcome. J Dent Res 86(12):1181-1186

5. Vanderveken OM, Hoekema A (2010) How to treat patients that do not tolerate continuous positive airway pressure. Breathe 7(2):157167

6. Marklund M, Verbraecken J, Randerath W (2012) Non-CPAP therapies in obstructive sleep apnoea: mandibular advancement device therapy. Eur Respir J 39(5):1241-1247

7. Hoekema A, Stegenga B, Wijkstra PJ, van der Hoeven JH, Meinesz AF, de Bont LGM (2008) Obstructive sleep apnea therapy. J Dent Res 87(9):882-887

8. Aarab G, Lobbezoo F, Hamburger HL, Naeije M (2011) Oral appliance therapy versus nasal continuous positive airway pressure in obstructive sleep apnea: a randomized, placebo-controlled trial. Respiration 81(5):411-419

9. Bradley TD, Floras JS (2009) Obstructive sleep apnoea and its cardiovascular consequences. Lancet 373(9657):82-93

10. Gotsopoulos H, Kelly JJ, Cistulli PA (2004) Oral appliance therapy reduces blood pressure in obstructive sleep apnea: a randomized, controlled trial. Sleep 27(5):934-941

11. Vanderveken OM, Dieltjens M, Wouters K, De Backer WA, Van de Heyning PH, Braem MJ (2013) Objective measurement of compliance during oral appliance therapy for sleep-disordered breathing. Thorax 68(1):91-96

12. Dieltjens M, Vanderveken OM, Heyning PH, Braem MJ (2012) Current opinions and clinical practice in the titration of oral appliances in the treatment of sleep-disordered breathing. Sleep Med Rev 16(2): $177-185$

13. Ribeiro de Almeida F (2011) Complexity and efficacy of mandibular advancement splints: understanding their mode of action. J Clin Sleep Med 7(5):447-448

14. Lettieri CJ, Paolino N, Eliasson AH, Shah AA, Holley AB (2011) Comparison of adjustable and fixed oral appliances for the treatment of obstructive sleep apnea. J Clin Sleep Med 7(5):439-445

15. Vanderveken OM, Devolder A, Marklund M, Boudewyns AN, Braem MJ, Okkerse W, Verbraecken JA, Franklin KA, De Backer WA, Van de Heyning PH (2008) Comparison of a custom-made and a thermoplastic oral appliance for the treatment of mild sleep apnea. Am J Respir Crit Care Med 178(2):197-202

16. Hoffstein V (2007) Review of oral appliances for treatment of sleepdisordered breathing. Sleep Breath 11(1):1-22

17. Tsuda H, Almeida FR, Masumi S, Lowe AA (2010) Side effects of boil and bite type oral appliance therapy in sleep apnea patients. Sleep Breath 14(3):227-232

18. Friedman M, Hamilton C, Samuelson CG, Kelley K, PearsonChauhan K, Taylor D, Taylor R, Maley A, Hirsch MA (2012) 
Compliance and efficacy of titratable thermoplastic versus custom mandibular advancement devices. Otolaryngol Head Neck Surg 147(2):379-386

19. Chan AS, Cistulli PA (2009) Oral appliance treatment of obstructive sleep apnea: an update. Curr Opin Pulm Med 15(1):591-596

20. Vanderveken OM, Van de Heyning PH, Braem MJ (2012) Response to "compliance and efficacy of titratable thermoplastic versus custom mandibular advancement devices" from Friedman $\mathrm{M}$ et al. Otolaryngol Head Neck Surg 147(3):599-600

21. Ahrens A, McGrath C, Hägg U (2010) Subjective efficacy of oral appliance design features in the management of obstructive sleep apnea: a systematic review. Am J Orthod Dentofacial Orthop 138(5):559-576

22. Bezzon OL, Mattos MGC, Ribero RF (1997) Surveying removable partial dentures: the importance of guiding planes and path of insertion for stability. J Prosthet Dent 78(4):412-416

23. Koolstra JH, van Eijden TM (1997) Dynamics of the human masticatory muscles during a jaw open-close movement. J Biomech 30(9):883-889

24. Tannous F, Steiner M, Shahin R, Kern M (2012) Retentive forces and fatigue resistance of thermoplastic resin clasps. Dent Mater 28(3):273-278
25. Koji K (2000) Wear in relation to friction - a review. Wear 241(2): $151-157$

26. Aarab G, Lobbezoo F, Hamburger HL, Naeije M (2010) Effects of an oral appliance with different mandibular protrusion positions at a constant vertical dimension on obstructive sleep apnea. Clin Oral Invest 14(3):339-345

27. Peck CC, Sooch AS, Hannam AG (2002) Forces resisting jaw displacement in relaxed humans: a predominantly viscous phenomenon. J Oral Rehabil 29(2):151-160

28. Johnston CD, Gleadhill IC, Cinnamond MJ, Gabbey J, Burden DJ (2002) Mandibular advancement appliances and obstructive sleep apnoea: a randomized clinical trial. Eur J Orthod 24(3):251-262

29. Martinez-Gomis J, Willaert E, Nogues L, Pascual M, Somoza M, Monasterio C (2010) Five years of sleep apnea treatment with a mandibular advancement device. Side effects and technical complications. Angle Orthod 80(1):30-36

30. Cohen-Levy J, Petelle B, Pinguet J, Limerat E, Fleury B (2012) Forces created by mandibular advancement devices in OSAS patients : A pilot study during sleep. Sleep Breath. doi:10.1007/s11325012-0765-4 